Article

\title{
Bifurcation Characteristic Research on the Load Vertical Vibration of a Hydraulic Automatic Gauge Control System
}

\author{
Yong Zhu ${ }^{1} \oplus$, Shengnan Tang ${ }^{1,2, *}$, Chuan Wang ${ }^{1,3, *}$, Wanlu Jiang ${ }^{4}$, Xiaoming Yuan ${ }^{2,4}$ and \\ Yafei Lei ${ }^{4, *(D)}$ \\ 1 National Research Center of Pumps, Jiangsu University, Zhenjiang 212013, China; zhuyong@ujs.edu.cn \\ 2 State Key Laboratory of Fluid Power and Mechatronic Systems, Zhejiang University, \\ Hangzhou 310027, China; yuanxiaoming@ysu.edu.cn \\ 3 School of Hydraulic Energy and Power Engineering, Yangzhou University, Yangzhou 225002, China \\ 4 Hebei Provincial Key Laboratory of Heavy Machinery Fluid Power Transmission and Control, \\ Yanshan University, Qinhuangdao 066004, China; wljiang@ysu.edu.cn \\ * Correspondence: 2111811013@stmail.ujs.edu.cn (S.T.); wangchuan@ujs.edu.cn (C.W.); \\ yafeilei@stmail.ysu.edu.cn (Y.L.); Tel.: +86-0511-88799918 (S.T. \& C.W.); +86-0335-8061729 (Y.L.)
}

Received: 28 August 2019; Accepted: 2 October 2019; Published: 10 October 2019

\begin{abstract}
As the core control system of a rolling mill, the hydraulic automatic gauge control (HAGC) system is key to ensuring a rolling process with high speed, high precision and high reliability. However, a HAGC system is typically a mechanical-electric-hydraulic coupling system with nonlinear characteristics. The vertical vibration of the load easily occurs during the working process, which seriously affects the stability of the system and the causes are difficult to determine. In this work, the theory and method of nonlinear dynamics were employed. The load vertical vibration model of the HAGC system was established. Then, the multi-scale method was utilized to solve the obtained model, and the singularity theory was further applied to derive the transition set. Moreover, the research object of this article focused on some nonlinear factors such as excitation force, elastic force and damping force. The effects of the above feature parameters on bifurcation behavior were emphatically explored. The bifurcation characteristic of the load vertical vibration of the HAGC system was revealed. The research results indicate that the bifurcation curves in each sub-region, divided by the transition set, possess their own topological structure. The changes of the feature parameters, such as the nonlinear stiffness coefficient, liquid column height, nonlinear damping coefficient, and external excitation have an influence on the vibration amplitude of the HAGC system. By reasonably adjusting the nonlinear stiffness coefficient to effectively avoid the resonance region, the stability of the system will be facilitated. Furthermore, this is conducive to the system's stability as it properly controls the size of the liquid column height of the hydraulic cylinder. The appropriate nonlinear damping coefficient can decrease the unstable area, which is beneficial to the stability of the system. However, large external excitation is not conducive to the stability of the system.
\end{abstract}

Keywords: flow control; vertical vibration; bifurcation characteristic; nonlinear dynamics; hydraulic automatic gauge control system; rolling mill

\section{Introduction}

In the metallurgical industry, the rolling mill represents a core piece of equipment. However, it has been found that there are always some parameter changes or disturbance factors during the rolling process, which may induce vibration in the rolling mill $[1,2]$. This may even cause the load roll system to produce vertical vibration with large amplitude, leading to production accidents such as steel-heaping, 
strip breakage, component damage and so on [3-5]. The hydraulic automatic gauge control (HAGC) system is considered the core control system of a rolling mill. The stability of the HAGC system is crucial to ensuring a rolling process with high speed, high precision and high reliability.

For a long time, many scholars and research institutions have explored the rolling mill's vibration characteristics, and rich results have been demonstrated. Zhong et al. [6] explored the mechanism of interfacial coupling and electromechanical coupling in the vibration of rolling mills. It was found that the various vibration phenomena of a high-speed rolling mill were related to various interactions of the rolling process. Wang et al. [7] conducted a series of studies on the control of plate thickness and plate shape, which laid a theoretical foundation for improving the control accuracy of the HAGC system. Chen et al. [8] studied the nonlinear parametric vibration of a four-roll cold strip mill and explored the influence of the deformation resistance of rolled piece on parametric resonance. The effects of rolling speed and tension on the nonlinear vibration of the rolling mill were investigated by Sun et al. [9], and the influence of external excitation frequency on system stability was analyzed. Liu et al. [10] researched the vertical, nonlinear parametric vibration characteristics of the mill roll system, and discussed the influence of parameters such as damping, stiffness and external excitation on system stability. In consideration of the change of parameters, Liu et al. [11] analyzed the partial bifurcation phenomenon of the load roll system, finding that both internal resonance and main resonance have an amplitude jump phenomenon. Yan et al. [12] studied the coupling vibration mechanism and vibration suppression method of the rolling mill. The characteristics and laws of vibration of the hot rolling mill were studied by the analysis of the measured signals, both in the time and frequency domain [13,14]. Yang et al. [15] researched the nonlinear modeling and stability of the vertical vibration of a cold rolling mill. The singular value theory was used to analyze the stability of the system. Moreover, the effects of parameters such as damping and stiffness on the vibration characteristics of the system were researched. In terms of periodic excitation, Bi et al. $[16,17]$ studied the bifurcation mechanism of oscillations of the dynamic system. Wang and Qian et al. [18,19] investigated the effects of important components such as pump [20-23] and valve [24,25] on the vibration characteristics. Bai et al. [26-28] researched the vibration of the pump under varied conditions. Zhang et al. [29-31] studied the influence of excitation forces on the vibration of the pump and the measure of noise reduction. Niziol et al. [32] investigated the effect of rolling speed on the vibration of a mill roll system, and analyzed the steady state domain of the vibration of the roller system under different rolling speeds. Heidari et al. [33,34] studied the influence of friction damping and lubrication state on the flutter of cold strip mill, and analyzed the effects of some main parameters of lubricant on the critical vibration velocity. In order to study the self-excited vibration and nonlinear paramagnetic vibration of the rolling process, a nonlinear mathematical model for the vibration of the rolling mill was established by Drzymala et al. [35].

The above research results have provided a theoretical guidance for the vibration mechanism analysis and vibration suppression of the rolling mill system. Nevertheless, based on the existing research results, some problems have been discovered. In most of the research results, the influence of the HAGC system is often overlooked when the vertical vibration of the rolling mill is analyzed [36,37]. In essence, the HAGC system is a nonlinear closed-loop system, which has many factors affecting its stability. If it is unstable, it will certainly have an influence on the vibration characteristics of the load roll system [38,39]. In addition, with the development of nonlinear science, it is of important theoretical significance to reveal the essence and mechanism of the system dynamic process by applying the nonlinear dynamics theory, which simultaneously presents broad application prospects [40-42]. This area is worthy of further study when the theory and method of nonlinear dynamics are utilized to explore the vibration mechanism of the HAGC system, and then to obtain the influence law and incentive of nonlinear factors on the vibration characteristics of the system.

In this paper, based on the theory and method of nonlinear dynamics, the influence of some nonlinear parameters of the HAGC system on load vertical vibration will be researched. Moreover, the bifurcation characteristics of load vertical vibration will be emphatically explored to reveal the instability mechanism of the HAGC system. In Section 2, the vertical vibration model of the load of the 
HAGC system is established. In Section 3, the bifurcation characteristic of load vertical vibration is analyzed. In Section 4, the effects of some feature parameters on bifurcation behavior are thoroughly explored. In Section 5, some conclusions are provided.

\section{Vertical Vibration Model of Load}

The external load of the HAGC system consists of several rollers, which present the symmetrical structure [43]. The basic load structure of the commonly used, four-high mill's HAGC system is shown in Figure 1.

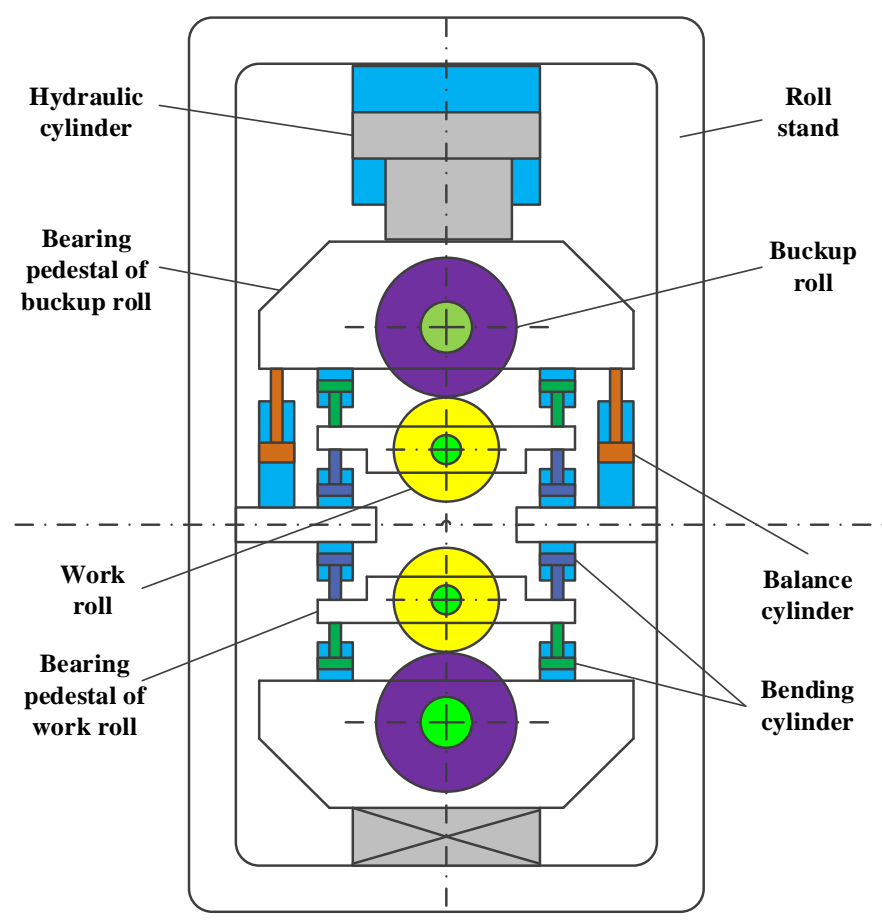

Figure 1. Structural schematic diagram of load.

In addition, the structure and vibration of load possess symmetry [44]. Moreover, some nonlinear factors such as nonlinear stiffness, nonlinear damping and nonlinear excitation are also considered. Then, a nonlinear load vertical vibration model of the HAGC system is built, as displayed in Figure 2 .

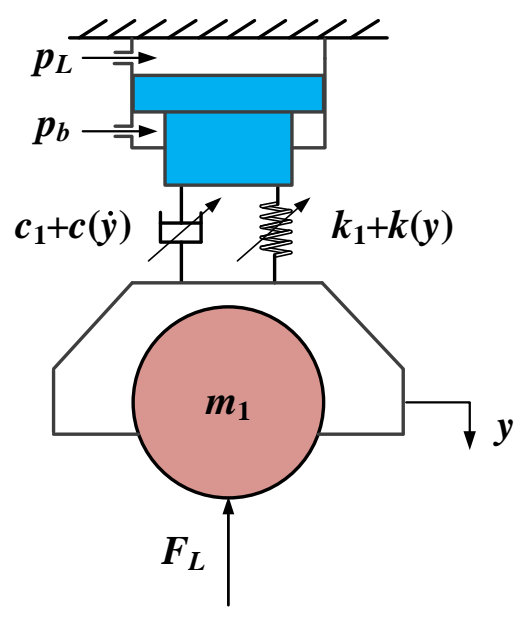

Figure 2. Vertical vibration model of load. 
According to Newton's second law, the nonlinear load vertical vibration equation of a HAGC system can be represented as:

$$
\begin{gathered}
m_{1} \ddot{y}+c_{1} \dot{y}+k_{1} y+\alpha F_{k}(y)+\beta F_{c}(\dot{y})=\Delta F \\
\Delta F=p_{L} A_{p}-p_{b} A_{b}-F_{L}
\end{gathered}
$$

where, $m_{1}$ is equivalent mass; $c_{1}$ and $k_{1}$ are linear damping coefficient and linear stiffness coefficient, respectively; $y$ is vibration displacement. $F_{k}(y)$ and $F_{c}(\dot{y})$ are nonlinear elastic force and nonlinear friction of hydraulic cylinder, respectively; $\alpha$ and $\beta$ are the action coefficient of nonlinear stiffness and nonlinear damping, respectively; $A_{p}$ and $A_{b}$ are the effective working area of rodless cavity and rod cavity, respectively; $p_{L}$ and $p_{b}$ are the working pressure of rodless cavity and rod cavity, respectively; $F_{L}$ is external load force. $\Delta F$ is external disturbance excitation. $\Delta F$ is mainly caused by the factors such as the pulsation of oil source, the fluctuations of rolled metal thickness or tension etc., which can be expressed by $F \cos \omega t$ [45]. $F$ is the amplitude of external excitation.

Among them, the expression of nonlinear elastic force $F_{k}(y)$ can be expressed by [46]:

$$
F_{k}(y)=\left(\frac{A_{p} \beta_{e}}{L_{1}}+\frac{A_{b} \beta_{e}}{L-L_{1}}\right) y+\left[\frac{A_{p} \beta_{e}}{L_{1}^{3}}+\frac{A_{b} \beta_{e}}{\left(L-L_{1}\right)^{3}}\right] y^{3}
$$

where $L_{1}$ is the liquid column height of control cavity of hydraulic cylinder.

The expression of nonlinear friction $F_{c}(\dot{y})$ can be displayed as [47,48]:

$$
F_{c}(\dot{y})=\left\{\begin{array}{cc}
F_{N}\left(\mu_{s}-\mu_{1} \dot{y}+\mu_{2} \dot{y}^{3}\right) & \dot{y}>0 \\
F & \dot{y}=0 \\
F_{N}\left(-\mu_{s}-\mu_{1} \dot{y}+\mu_{2} \dot{y}^{3}\right) & \dot{y}<0
\end{array}\right.
$$

where

$$
\begin{gathered}
\mu_{1}=\frac{3\left(\mu_{s}-\mu_{m}\right)}{2 v_{m}} \\
\mu_{2}=\frac{\mu_{s}-\mu_{m}}{2 v_{m}^{3}}
\end{gathered}
$$

where $\mu_{m}$ is the maximum dynamic friction factor; $\mu_{s}$ is static friction factor; $v_{m}$ is vibration velocity; $F_{N}$ is the positive pressure of piston acts on cylinder wall, which mainly depends on the factors such as the tightness degree of assembly, the hardness of sealing material, and the radial component of load.

\section{Analysis of Bifurcation Characteristic}

As below, the bifurcation characteristic of load vertical vibration is analyzed in detail. Moreover, the multi-scale method is utilized to solve the nonlinear equation of load vertical vibration.

Firstly, the small parameter factor $\varepsilon$ and a series of slow-change time scales $T_{n}$ are employed $[49,50]$.

$$
T_{n}=\varepsilon^{n} t \quad n=0,1,2 \cdots
$$

Then, $T_{n}$ are regarded as independent variables; there are:

$$
\begin{gathered}
\frac{\mathrm{d}}{\mathrm{d} t}=\frac{\partial}{\partial T_{0}}+\varepsilon \frac{\partial}{\partial T_{1}}+\cdots+\varepsilon^{n} \frac{\partial}{\partial T_{n}}=D_{0}+\varepsilon D_{1}+\cdots+\varepsilon^{n} D_{n} \\
=\frac{\mathrm{d}}{\mathrm{d} t}\left(\frac{\partial}{\partial T_{0}}+\varepsilon \frac{\partial}{\partial T_{1}}+\cdots+\varepsilon^{n} \frac{\partial}{\partial T_{n}}\right) \\
=\left(D_{0}+\varepsilon D_{1}+\cdots+\varepsilon^{n} D_{n}\right)^{2} \\
=D_{0}^{2}+2 \varepsilon D_{0} D_{1}+\varepsilon^{2}\left(D_{1}^{2}+2 D_{0} D_{2}\right)+\cdots
\end{gathered}
$$


where $D_{n}$ is a symbol of partial differential operator:

$$
D_{n}=\frac{\partial}{\partial T_{n}} \quad n=0,1,2 \cdots
$$

Equation (1) can be further sorted as:

$$
\ddot{y}+\omega_{0}^{2} y=\frac{1}{m_{1}}\left[F \cos (\omega t)-\alpha F_{k}(y)-\beta F_{c}(\dot{y})-c_{1} \dot{y}\right]
$$

where $\omega_{0}=\sqrt{k_{1} / m_{1}}$.

Then, the right side of Equation (11) is multiplied by $\varepsilon$; there is:

$$
\ddot{y}+\omega_{0}^{2} y=\frac{\varepsilon}{m_{1}}\left[F \cos (\omega t)-\alpha F_{k}(y)-\beta F_{c}(\dot{y})-c_{1} \dot{y}\right]
$$

Suppose that $\omega$ is close to $\omega_{0}$, that is:

$$
\omega=\omega_{0}+\varepsilon \sigma
$$

where $\omega$ is perturbation frequency, $\omega_{0}$ is natural frequency, $\sigma$ is the frequency tuning factor.

Equation (13) is substituted into Equation (12); there is:

$$
\ddot{y}+\omega_{0}^{2} y=\varepsilon\left[\frac{F}{m_{1}} \cos \left(\omega_{0}+\varepsilon \sigma\right) t+f(y, \dot{y})\right]
$$

Among them:

$$
f(y, \dot{y})=-\frac{1}{m_{1}}\left[\alpha F_{k}(y)+\beta F_{c}(\dot{y})+c_{1} \dot{y}\right]
$$

Assume that Equation (14) has the solution with the following form when it is under the external excitation:

$$
y=y_{0}\left(T_{0}, T_{1}\right)+y_{1}\left(T_{0}, T_{1}\right)
$$

Substituting Equation (16) into Equation (14), Equations (8) and (9) are introduced. Then, the partial differential equations can be obtained:

$$
\left\{\begin{array}{l}
D_{0}^{2} y_{0}+\omega_{0}^{2} y_{0}=0 \\
D_{0}^{2} y_{1}+\omega_{0}^{2} y_{1}=-2 D_{0} D_{1} y_{0}+f\left(y_{0}, D_{0} y_{0}\right)+\frac{F}{m_{1}} \cos \left(\omega_{0} T_{0}+\sigma T_{1}\right)
\end{array}\right.
$$

The solution for the first equation of Equation (17) is set as:

$$
\begin{gathered}
y_{0}\left(T_{0}, T_{1}\right)=a\left(T_{1}\right) \cos \left[\omega_{0} T_{0}+\psi\left(T_{1}\right)\right]=A\left(T_{1}\right) e^{\mathrm{i} \omega_{0} T_{0}}+\mathrm{cc} \\
A\left(T_{1}\right)=\frac{a\left(T_{1}\right)}{2} e^{\mathrm{i} \psi\left(T_{1}\right)}
\end{gathered}
$$

where $a\left(T_{1}\right)$ and $\psi\left(T_{1}\right)$ are the slow-change functions of vibration amplitude and phase angle, respectively. cc indicates the conjugated plural.

Substituting Equation (18) into the second equation of Equation (17), there is:

$$
\begin{gathered}
D_{0}^{2} y_{1}+\omega_{0}^{2} y_{1}=\mathrm{i} \omega_{0}\left(-2 D_{1}-\frac{c_{1}}{m_{1}}\right) A \mathrm{e}^{\mathrm{i} \omega_{0} T_{0}}+\frac{F}{2 m_{1}} \mathrm{e}^{\mathrm{i}\left(\omega_{0} T_{0}+\sigma T_{1}\right)} \\
-\frac{\alpha \beta_{e}}{m_{1}}\left[\left(\frac{A_{p}}{L_{1}}+\frac{A_{b}}{L-L_{1}}\right) A \mathrm{e}^{\mathrm{i} \omega_{0} T_{0}}+\left(\frac{A_{p}}{L_{1}^{3}}+\frac{A_{b}}{\left(L-L_{1}\right)^{3}}\right)\left(A^{3} \mathrm{e}^{\mathrm{i} 3 \omega_{0} T_{0}}+3 A^{2} \bar{A} \mathrm{e}^{\mathrm{i} \omega_{0} T_{0}}\right)\right] \\
-\frac{\beta F_{N}}{m_{1}}\left[\mu_{S} \operatorname{sgn}(\dot{y})-\mathrm{i} \omega_{0} \mu_{1} A \mathrm{e}^{\mathrm{i} \omega_{0} T_{0}}-\mathrm{i} \omega_{0}^{3} \mu_{2}\left(A^{3} \mathrm{e}^{\mathrm{i} 3 \omega_{0} T_{0}}-3 A^{2} \bar{A} \mathrm{e}^{\mathrm{i} \omega_{0} T_{0}}\right)\right]+\mathrm{cc}
\end{gathered}
$$


The secular term is further eliminated. In Equation (20), the coefficient of $\mathrm{e}^{\mathrm{i} \omega_{0} T_{0}}$ is set to zero; there is:

$$
\begin{gathered}
\mathrm{i} \omega_{0}\left(2 D_{1}+\frac{c_{1}}{m_{1}}\right) A-\frac{F}{2 m_{1}} \mathrm{e}^{\mathrm{i} \sigma T_{1}}+\frac{\alpha \beta_{e}}{m_{1}}\left[\left(\frac{A_{p}}{L_{1}}+\frac{A_{b}}{L-L_{1}}\right) A+\left(\frac{A_{p}}{L_{1}^{3}}+\frac{A_{b}}{\left(L-L_{1}\right)^{3}}\right)\left(3 A^{2} \bar{A}\right)\right] \\
+\frac{\beta F_{N}}{m_{1}}\left[-\mathrm{i} \omega_{0} \mu_{1} A+\mathrm{i} 3 \omega_{0}^{3} \mu_{2} A^{2} \bar{A}\right]=0
\end{gathered}
$$

Then, Equation (19) is substituted into Equation (21). Moreover, the real and imaginary parts are separated; there are:

$$
\left\{\begin{array}{l}
D_{1} a=-\frac{c_{1}}{2 m_{1}} a+\frac{\beta F_{N}}{m_{1}}\left(\frac{\mu_{1}}{2} a-\frac{9 a^{3}}{8} \omega_{0}^{2} \mu_{2}\right)+\frac{F}{2 m_{1} \omega_{0}} \sin \left(\sigma T_{1}-\psi\right) \\
D_{1} \psi=\frac{\alpha \beta_{e}}{2 m_{1} \omega_{0}}\left[\left(\frac{A_{p}}{L_{1}}+\frac{A_{b}}{L-L_{1}}\right)+\frac{3 a^{2}}{4}\left(\frac{A_{p}}{L_{1}^{3}}+\frac{A_{b}}{\left(L-L_{1}\right)^{3}}\right)\right]-\frac{F}{2 m_{1} \omega_{0} a} \cos \left(\sigma T_{1}-\psi\right)
\end{array}\right.
$$

Suppose that $\varphi=\sigma T_{1}-\psi$, then Equation (22) can be changed into:

$$
\left\{\begin{array}{l}
D_{1} a=-\frac{c_{1}}{2 m_{1}} a+\frac{\beta F_{N}}{m_{1}}\left(\frac{\mu_{1}}{2} a-\frac{9 a^{3}}{8} \omega_{0}^{2} \mu_{2}\right)+\frac{F}{2 m_{1} \omega_{0}} \sin \varphi \\
D_{1} \varphi=\sigma-\frac{\alpha \beta_{e}}{2 m_{1} \omega_{0}}\left[\left(\frac{A_{p}}{L_{1}}+\frac{A_{b}}{L-L_{1}}\right)+\frac{3 a^{2}}{4}\left(\frac{A_{p}}{L_{1}^{3}}+\frac{A_{b}}{\left(L-L_{1}\right)^{3}}\right)\right]+\frac{F}{2 m_{1} \omega_{0} a} \cos \varphi
\end{array}\right.
$$

In Equation (23), when $D_{1} a=0$ and $D_{1} \varphi=0$, the system has a stable vibration amplitude and frequency; there are:

$$
\left\{\begin{array}{l}
\frac{c_{1}}{m_{1}} a-\frac{\beta F_{N} a}{m_{1}}\left(\mu_{1}-\frac{9 a^{2}}{4} \omega_{0}^{2} \mu_{2}\right)=\frac{F}{m_{1} \omega_{0}} \sin \varphi \\
2 \sigma a-\frac{\alpha \beta_{e} a}{m_{1} \omega_{0}}\left[\left(\frac{A_{p}}{L_{1}}+\frac{A_{b}}{L-L_{1}}\right)+\frac{3 a^{2}}{4}\left(\frac{A_{p}}{L_{1}^{3}}+\frac{A_{b}}{\left(L-L_{1}\right)^{3}}\right)\right]=-\frac{F}{m_{1} \omega_{0}} \cos \varphi
\end{array}\right.
$$

In Equation (24), the square sum is performed to eliminate the parameter $\varphi$; there are:

$$
\begin{gathered}
\left\{\frac{c_{1}}{m_{1}} a-\frac{\beta F_{N} a}{m_{1}}\left(\mu_{1}-\frac{9 a^{2}}{4} \omega_{0}^{2} \mu_{2}\right)\right\}^{2}+ \\
\left\{2 \sigma a-\frac{\alpha \beta_{e} a}{m_{1} \omega_{0}}\left[\left(\frac{A_{p}}{L_{1}}+\frac{A_{b}}{L-L_{1}}\right)+\frac{3 a^{2}}{4}\left(\frac{A_{p}}{L_{1}^{3}}+\frac{A_{b}}{\left(L-L_{1}\right)^{3}}\right)\right]\right\}^{2}=\left(\frac{F}{m_{1} \omega_{0}}\right)^{2}
\end{gathered}
$$

Equation (25) is unfolded and organized; there is:

$$
a^{6}+\lambda_{1} a^{4}+\lambda_{2} a^{2}+\mu=0
$$

Among them:

$$
\begin{gathered}
\lambda_{1}=-\left\{\frac{\alpha \beta_{e}}{3 \omega_{0}}\left[\frac{A_{p}}{L_{1}^{3}}+\frac{A_{b}}{\left(L-L_{1}\right)^{3}}\right]\left[m_{1} \sigma-\frac{\alpha \beta_{e}}{2 \omega_{0}}\left(\frac{A_{p}}{L_{1}}+\frac{A_{b}}{L-L_{1}}\right)\right]-\frac{\beta F_{N} \mu_{2} \omega_{0}^{2}}{2}\left(c_{1}-\beta F_{N} \mu_{1}\right)\right\} \\
\div\left\{\frac{\alpha^{2} \beta_{e}^{2}}{16 \omega_{0}^{2}}\left[\frac{A_{p}}{L_{1}^{3}}+\frac{A_{b}}{\left(L-L_{1}\right)^{3}}\right]^{2}+\frac{9 \beta^{2} F_{N}^{2} \mu_{2}^{2} \omega_{0}^{4}}{16}\right\} \\
\left.\lambda_{2}=\left\{\left[2 \sigma-\frac{\alpha \beta_{e}}{m_{1} \omega_{0}}\left(\frac{A_{p}}{L_{1}}+\frac{A_{b}}{L-L_{1}}\right)\right]^{2}+\frac{c 1}{m 1}-\frac{\beta F_{N} \mu_{1}}{m 1}\right)^{2}\right\} \\
\div\left\{\frac{9 \alpha^{2} \beta_{e}^{2}}{16 m_{1}^{2} \omega_{0}^{2}}\left[\frac{A_{p}}{L_{1}^{3}}+\frac{A_{b}}{\left(L-L_{1}\right)^{3}}\right]^{2}+\frac{81 \beta^{2} F_{N}^{2} \mu_{2}^{2} \omega_{0}^{4}}{16 m_{1}^{2}}\right\} \\
\mu=-\left(\frac{F}{m_{1}}\right)^{2} \div\left\{\frac{9 \alpha^{2} \beta_{e}^{2}}{16 m_{1}^{2}}\left[\frac{A_{p}}{L_{1}^{3}}+\frac{A_{b}}{\left(L-L_{1}\right)^{3}}\right]^{2}+\frac{81 \beta^{2} F_{N}^{2} \mu_{2}^{2} \omega_{0}^{6}}{16 m_{1}^{2}}\right\}
\end{gathered}
$$

Equation (26) can be further written as:

$$
a^{7}+\lambda_{1} a^{5}+\lambda_{2} a^{3}+\mu a=0
$$


According to the singularity theory [51], Equation (30) is the universal unfolding of the paradigm $a^{7}+\mu a=0, \lambda_{1}$ and $\lambda_{2}$ are the open fold parameters, and $\mu$ is the external disturbance quantity. If the values of the open fold parameters are different, the system will have different bifurcation patterns.

Assume:

$$
G(a, \mu, \lambda)=a^{7}+\lambda_{1} a^{5}+\lambda_{2} a^{3}+\mu a
$$

Then, the derivations about $a$ and $\mu$ in Equation (31) are respectively performed, and the following can be obtained:

$$
\begin{gathered}
\dot{G}_{a}(a, \mu, \lambda)=7 a^{6}+5 \lambda_{1} a^{4}+3 \lambda_{2} a^{2}+\mu \\
\dot{G}_{\mu}(a, \mu, \lambda)=a \\
\ddot{G}_{a}(a, \mu, \lambda)=42 a^{5}+20 \lambda_{1} a^{3}+6 \lambda_{2} a
\end{gathered}
$$

In the singularity theory, the transition set is a very important concept. This is the set of folding parameters corresponding to the non-persistent bifurcation diagram of the universal unfolding $G(a, \mu, \lambda)$. The sets of bifurcation point, lag point, and double limit point correspond to the three types of the non-persistence bifurcation diagram [52,53].

(1) Bifurcation point set

$B=\left\{\lambda \in \mathbf{R}^{n}\right.$ exist $(a, \mu) \in \mathbf{R} \times \mathbf{R}$, make $G=\dot{G}_{a}=\dot{G}_{\mu}=0$ at $\left.(a, \mu, \lambda)\right\}$;

(2) Lag point set

$H=\left\{\lambda \in \mathbf{R}^{n} \mid\right.$ exist $(a, \mu) \in \mathbf{R} \times \mathbf{R}$, make $G=\dot{G}_{a}=\ddot{G}_{a}=0$ at $\left.(a, \mu, \lambda)\right\}$;

(3) Double limit point set

$D=\left\{\lambda \in \mathbf{R}^{n} \mid\right.$ exist $\left(a_{i}, \mu\right)(i=1,2) \in \mathbf{R} \times \mathbf{R}, a_{1} \neq a_{2}$, make $G=\dot{G}_{a}=0$ at $\left.\left(a_{i}, \mu, \lambda\right)\right\}$;

(4) Transition set: $\sum=B \cup H \cup D$

The transition set can divide the neighborhood of the origin of the real space $\mathbf{R}^{n}$ into several subregions, and the bifurcation diagram of the universal unfolding $G(a, \mu, \lambda)$ in each subregion is persistent. In the same subregion, the bifurcation diagrams corresponding to different folding parameters $\lambda$ are equivalent. Then, all the persistent bifurcation maps of $G(a, \mu, \lambda)$ can be enumerated according to these subregions.

Next, singularity theory is applied to solve the transition set of bifurcation Equation (30), and the bifurcation characteristics of the system are explored. The subscripts $R$ and $I$ represent the new transition set generated by the conventional transition set and nonlinear action, respectively [54].

Bifurcation point set:

$$
B_{R}=\varnothing \quad B_{I}=\varnothing
$$

Lag point set:

$$
H_{R}=\left\{\frac{3 \lambda_{2}^{2}}{\lambda_{1}^{2}}-\lambda_{2}=0\right\} \quad H_{I}=\varnothing
$$

Double limit point set:

$$
D_{R}=\varnothing \quad D_{I}=\left\{6 a^{4}+4 \lambda_{1} a^{2}+2 \lambda_{2}=0\right\}
$$

Transition set:

$$
\sum=B_{R} \cup H_{R} \cup D_{R} \cup B_{I} \cup H_{I} \cup D_{I}
$$

At this point, the transition set of the system under the open fold parameters $\lambda_{1}$ and $\lambda_{2}$ is shown in Figure 3. The topological structures of the bifurcation curves in different subregions divided by the transition set are displayed in Figure 4. 


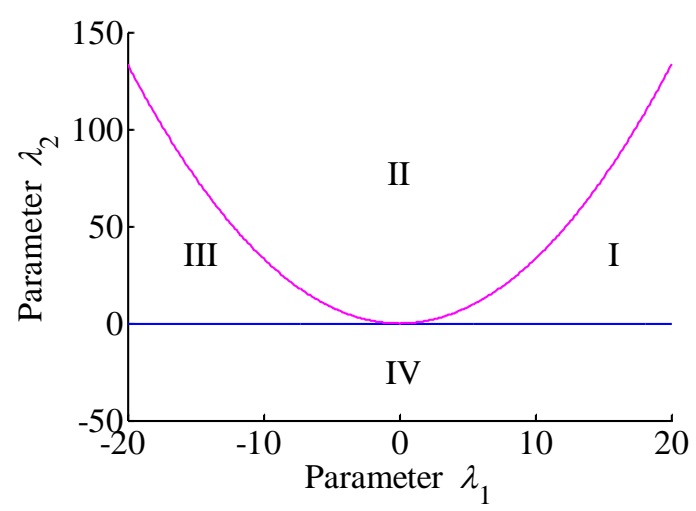

Figure 3. Transition set.

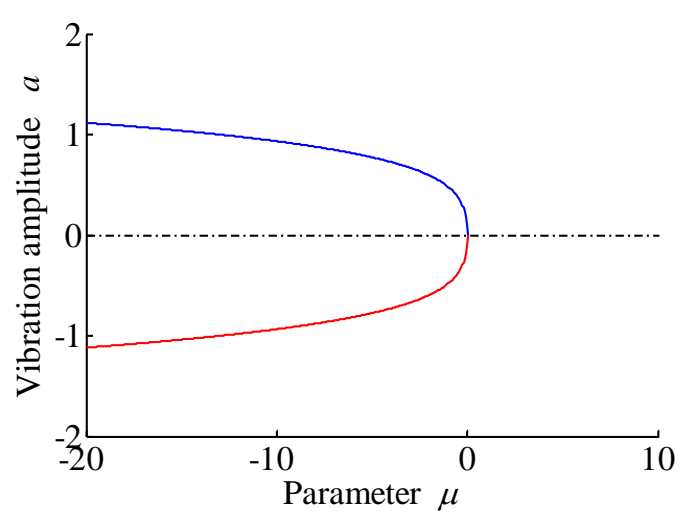

(a) region $\mathrm{I}$

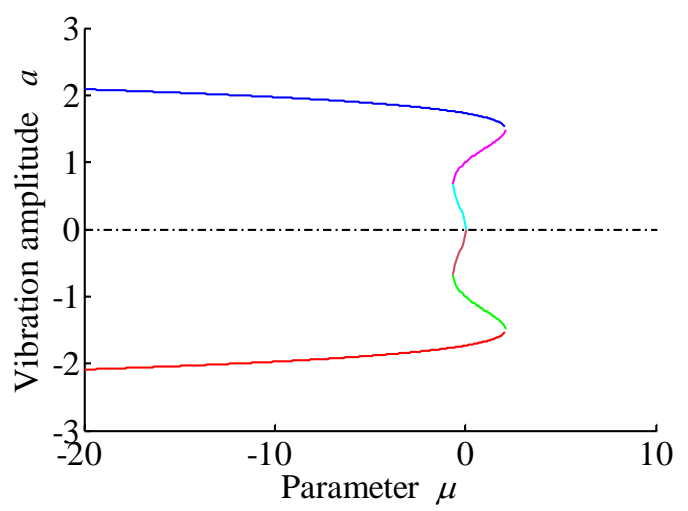

(c) region III

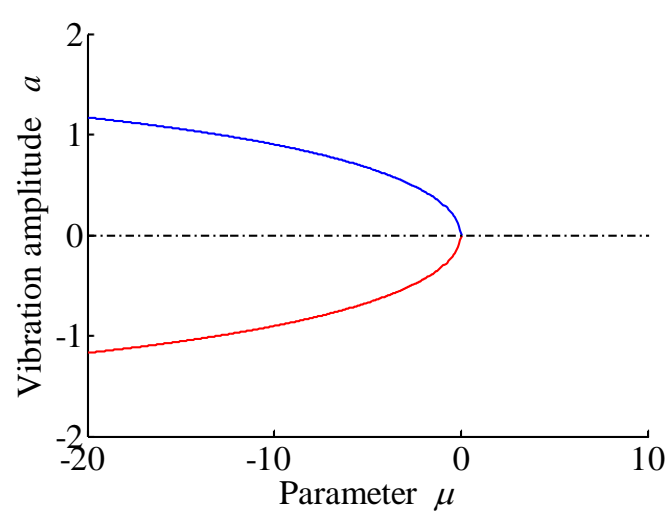

(b) region II

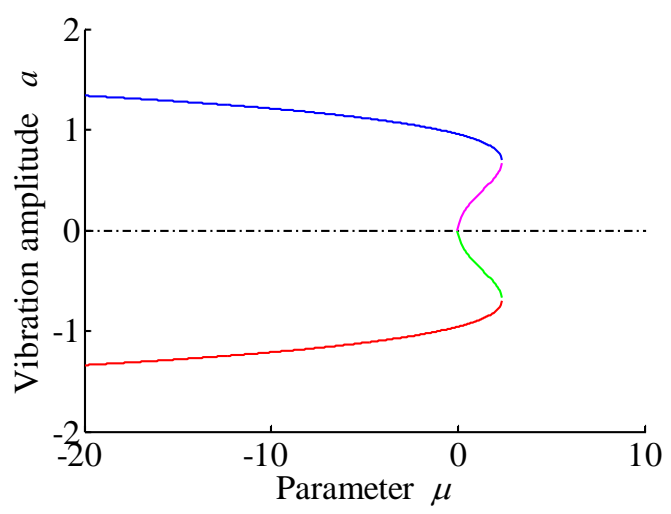

(d) region IV

Figure 4. Topological structure of bifurcation curves in different subregions.

According to Figures 3 and 4, the system plane is divided into four subregions (I, II, III, IV) by the transition set under the open fold parameters $\lambda_{1}$ and $\lambda_{2}$. The bifurcation curves in each subregion have their own topological structure. Furthermore, with the change of $\lambda_{1}$ and $\lambda_{2}$, the topological structure changes at the transition set. It indicates that the system has different vibration behaviors in diverse subregions and will exhibit different bifurcation behaviors under various parameter combinations. Therefore, by analyzing the bifurcation characteristics of the system, the parameter region that causes the system to be unstable can be determined. Meanwhile, from the obtained topological structures of the bifurcation curve in different subregions, the bifurcation state of the system can be changed by the disturbance parameter $\mu$. In a certain bifurcation form, the change of $\mu$ will lead to the change of vibration amplitude, which in turn changes the vibration behavior of the system. 


\section{Research on Bifurcation Behavior}

The load of the HAGC system will be affected by different forces during the working process. The influence factors are diverse and complex. The research object of this article will focus on some nonlinear factors such as the excitation force, elastic force and damping force. The selected nonlinear forces are important influence factors. The effects of the above feature parameters on bifurcation behavior are explored.

The actual physical parameters of the 650 4/6-roll cold rolling mill from the "National Engineering Research Center for Equipment and Technology of Cold Strip Rolling" are employed in the following research. Some unmeasured parameters are empirical estimates. The parameters are shown in Table 1. The photos of the 650 4/6-roll cold rolling mill are displayed in Figure 5.

Table 1. Parameters of the numerical experiment.

\begin{tabular}{|c|c|c|c|c|c|}
\hline Physical Quantity & Value & Unit & Physical Quantity & Value & Unit \\
\hline equivalent mass $m_{1}$ & 8656 & $\mathrm{~kg}$ & bulk modulus $\beta_{e}$ & $780 \times 10^{6}$ & $\mathrm{~Pa}$ \\
\hline $\begin{array}{c}\text { equivalent linear stiffness } \\
\text { coefficient } k_{1}\end{array}$ & $1.6724 \times 10^{9}$ & $\mathrm{~N} / \mathrm{m}$ & dynamic friction factor $\mu_{m}$ & 0.01 & - \\
\hline $\begin{array}{l}\text { equivalent linear damping } \\
\text { coefficient } c_{1}\end{array}$ & $1.2923 \times 10^{5}$ & $\mathrm{~N} \cdot \mathrm{s} / \mathrm{m}$ & static friction factor $\mu_{S}$ & 0.02 & - \\
\hline total stroke $L$ & 260 & $\mathrm{~mm}$ & vibration velocity $v_{m}$ & 0.01 & $\mathrm{~m} / \mathrm{s}$ \\
\hline effective working area $A_{p}$ & $19.635 \times 10^{-2}$ & $\mathrm{~m}^{2}$ & positive pressure $F_{N}$ & $0.04 \times 10^{6}$ & $\mathrm{~N}$ \\
\hline effective working area $A_{b}$ & $3.0159 \times 10^{-2}$ & $\mathrm{~m}^{2}$ & oil density $\rho$ & 872 & $\mathrm{~kg} / \mathrm{m}^{3}$ \\
\hline
\end{tabular}

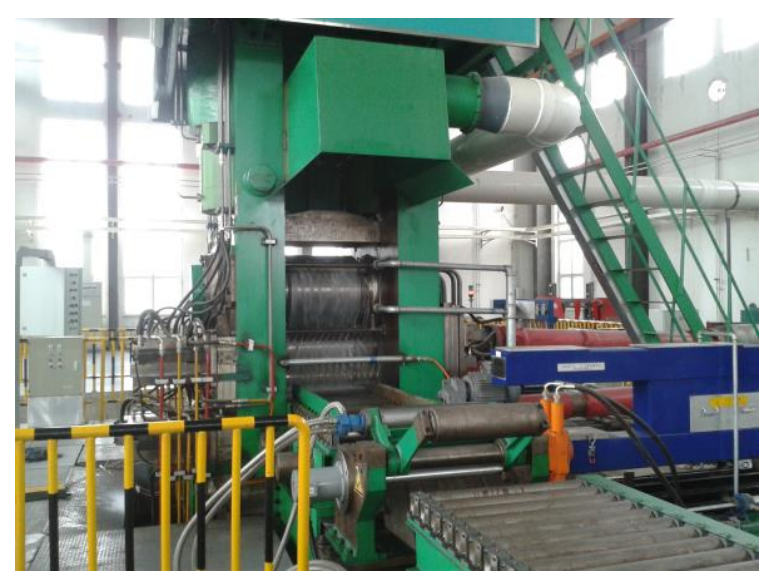

(a)

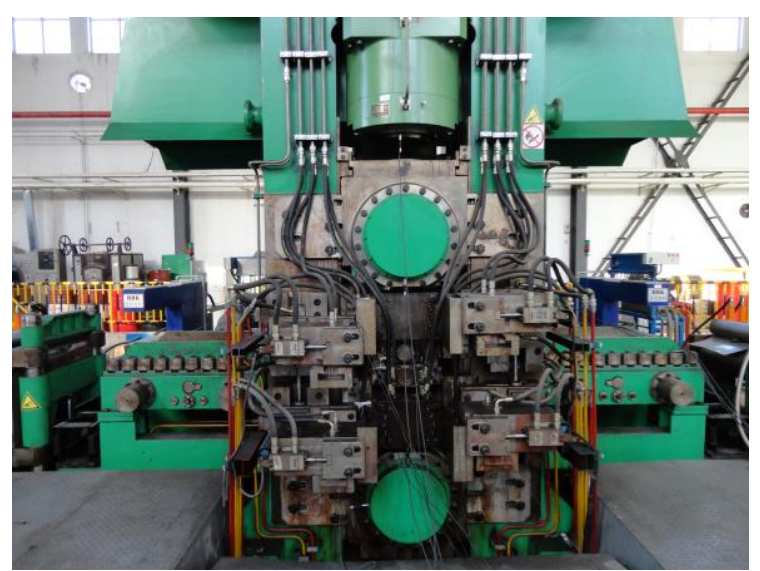

(b)

Figure 5. Photos of the 650 4/6-roll cold rolling mill.

The influence of nonlinear elastic force can be reflected by the nonlinear stiffness coefficient $\alpha$. Hence, the effect of $\alpha$ on bifurcation characteristics is firstly researched. According to Equation (11) derived in the previous section, the bifurcation diagram when $\alpha$ changes is revealed in Figure 6. The jump phenomenon of the vibration amplitude will gradually be enhanced with the increase in $\alpha$. The degree of jump phenomenon is especially severe in the resonance region. However, when it is far away from the resonance region, the degree of jump will be reduced. The primary reason for the above result is that the change of the nonlinear stiffness coefficient $\alpha$ affects the natural frequency $\omega_{0}$ of the system. As $\alpha$ increases, the natural frequency of the system increases. The change of natural frequency results in the resonance phenomenon when natural frequency couples with external excitation frequency $\omega$. However, the resonance phenomenon can cause the increase in system instability. Therefore, if $\alpha$ is reasonably adjusted to effectively avoid the resonance region, the stability of the system will be facilitated. 


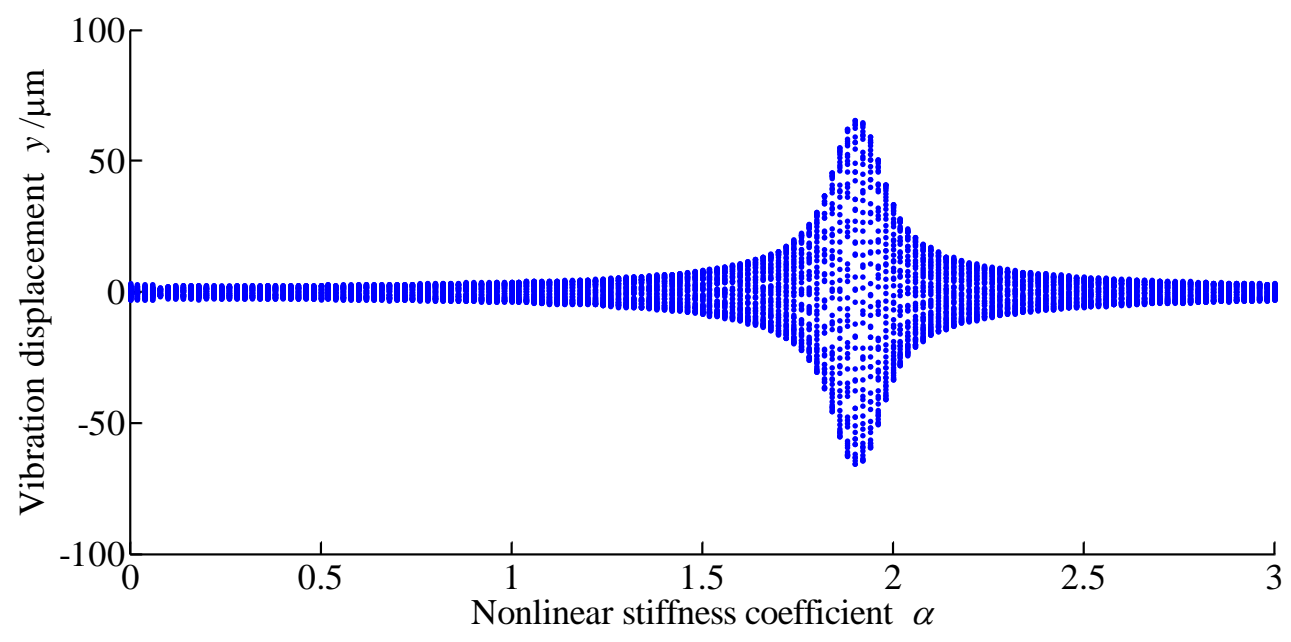

Figure 6. Bifurcation diagram with the variation of $\alpha$.

Additionally, the nonlinear elastic force can be influenced by the liquid column height $L_{1}$ of control cavity. So, the effect of $L_{1}$ on bifurcation characteristics was further studied. The bifurcation diagram with the variation of $L_{1}$ is displayed in Figure 7 . When $L_{1}$ is close to the two ends of hydraulic cylinder, the jump phenomenon of the vibration amplitude is more serious. When $L_{1}$ is away from the ends of the hydraulic cylinder, the degree of amplitude jump is relatively reduced. The bifurcation phenomenon near the middle position $(130 \mathrm{~mm})$ is more complex. The foremost reason is that the stiffness of the hydraulic spring is related to the piston position of the hydraulic cylinder. When the piston is in the middle position, the liquid compressibility is most affected. At this time, the hydraulic spring stiffness is small and the natural frequency of the system is low. Therefore, it shows poor stability in the system. Hence, properly controlling the size of $L_{1}$ is conducive to the stability of the system.

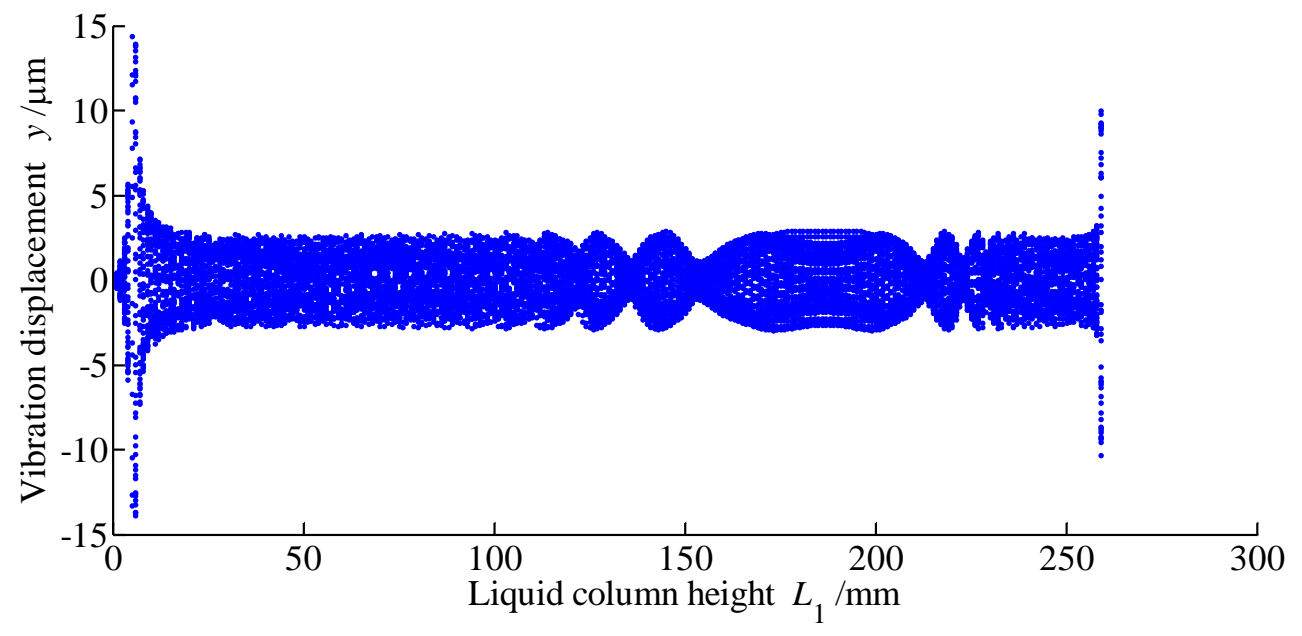

Figure 7. Bifurcation diagram with the variation of $L_{1}$.

The effect of nonlinear damping force can be reflected by the nonlinear damping coefficient $\beta$. Hence, the influence of $\beta$ on bifurcation characteristics was analyzed. The bifurcation diagram with the change of $\beta$ is illustrated in Figure 8. As can be observed, the jump phenomenon of the vibration amplitude will gradually decrease with the increase in $\beta$, and the vibration amplitude is effectively suppressed. However, when the value of $\beta$ exceeds a certain threshold, the suppression effect for vibration amplitude is no longer obvious, and the jump phenomenon of vibration amplitude still exists. The main reason is that the appropriate $\beta$ can narrow the frequency band of resonance and decrease the unstable area, which is beneficial to the stability of the system. 


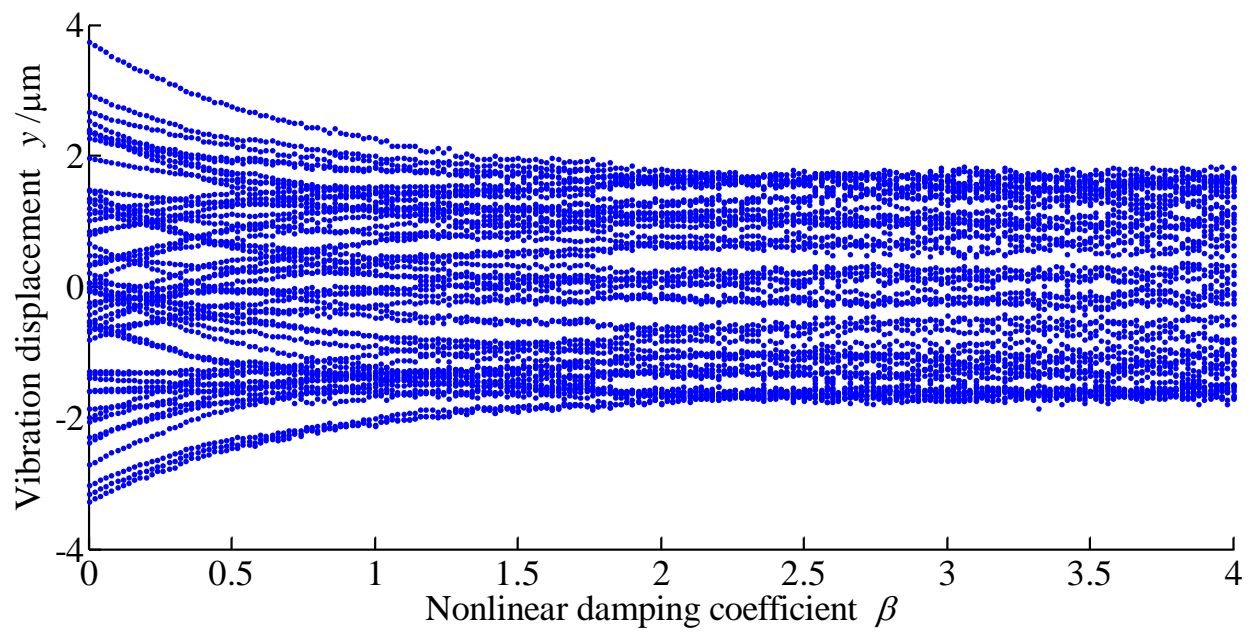

Figure 8. Bifurcation diagram with the variation of $\beta$.

Since the influence of nonlinear excitation force can be reflected by the external excitation amplitude $F$, the effect of $F$ on bifurcation characteristics was also investigated. The bifurcation diagram with the variation of $F$ is demonstrated in Figure 9. It will be observed that the jump phenomenon of vibration amplitude will gradually strengthen with the increase in $F$. Furthermore, the degree of jump will gradually increase. The main reason is that the increase in $F$ can widen the frequency band of resonance and augment the instability of the system.

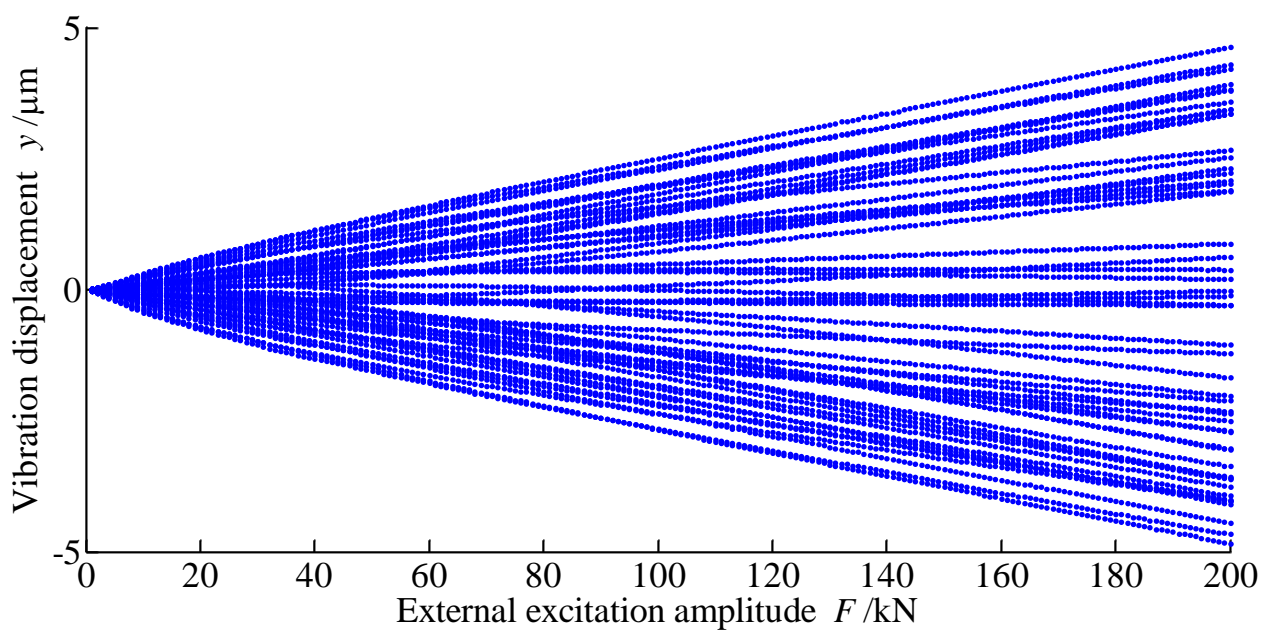

Figure 9. Bifurcation diagram with the variation of $F$.

\section{Conclusions}

On the basis of the theory of nonlinear dynamics, the bifurcation characteristics of load vertical vibration of the HAGC system were researched. The effects of some important parameters on bifurcation characteristics were emphatically explored. Through in-depth research, some conclusions are drawn:

(1) The bifurcation curves in each subregion have their own topological structure. With the change of the open fold parameters, the topological structure changes at the transition set. Moreover, the system has different vibration behaviors in diverse subregions, and will exhibit different bifurcation behaviors under various parameter combinations. Therefore, by analyzing the bifurcation characteristics of the system, the parameter region that causes the system to be unstable can be determined.

(2) With the increase in the nonlinear stiffness coefficient $\alpha$, the jump phenomenon of the vibration amplitude will gradually be enhanced. Especially, the degree of jump phenomenon is severe in the 
resonance region. However, the degree of jump will be reduced when it is far away from the resonance region. Therefore, if $\alpha$ is reasonably adjusted to effectively avoid the resonance region, the stability of the system will be facilitated.

(3) The jump phenomenon of the vibration amplitude is more serious when the liquid column height $L_{1}$ is close to the two ends of hydraulic cylinder. The degree of amplitude jump is relatively reduced when $L_{1}$ is located in the middle section of the hydraulic cylinder. However, the bifurcation phenomenon near the middle position is more complex. Hence, properly controlling the size of $L_{1}$ is conducive to the stability of the system.

(4) With the increase in the nonlinear damping coefficient $\beta$, the jump phenomenon of the vibration amplitude will gradually decrease, and the vibration amplitude is effectively suppressed. However, the suppression effect for vibration amplitude is no longer obvious when the value of $\beta$ exceeds a certain threshold, and the jump phenomenon of the vibration amplitude still exists. The appropriate $\beta$ can narrow the frequency band of resonance and decrease the unstable area, which is beneficial to the stability of the system.

(5) With the increase in the external excitation amplitude $F$, the jump phenomenon of the vibration amplitude will gradually strengthen. Moreover, the degree of jump will gradually increase, which is not conducive to the stability of the system.

The acquired results provide a theoretical basis for vibration traceability and suppression of the HAGC system. This research can provide an important basis for the further study on nonlinear dynamic behaviors of the HAGC system.

Author Contributions: Conceptualization, Y.Z. and W.J.; Methodology, S.T.; Investigation, Y.Z. and S.T.; Writing-Original Draft Preparation, Y.Z.; Writing—Review \& Editing, X.Y. and Y.L.; Supervision, C.W.

Funding: This research was funded by National Natural Science Foundation of China (No. 51805214, 51875498), China Postdoctoral Science Foundation (No. 2019M651722), Natural Science Foundation of Hebei Province (No. E2018203339, E2017203129), Nature Science Foundation for Excellent Young Scholars of Jiangsu Province (No. BK20190101), Open Foundation of National Research Center of Pumps, Jiangsu University (No. NRCP201604) and Open Foundation of the State Key Laboratory of Fluid Power and Mechatronic Systems (No. GZKF-201820).

Conflicts of Interest: The authors declare no conflict of interest.

\section{References}

1. Zheng, Y.J.; Li, Y.G.; Shen, G.X.; Xie, M.L. Spatial vibration characteristics of six-high cold strip rolling mills. Ironmak. Steelmak. 2017, 45, 1-6. [CrossRef]

2. Shao, Y.; Deng, X.; Yuan, Y.; Mechefske, C.K.; Chen, Z. Characteristic recognition of chatter mark vibration in a rolling mill based on the non-dimensional parameters of the vibration signal. J. Mech. Sci. Technol. 2014, 28, 2075-2080. [CrossRef]

3. Wu, S.; Shao, Y.; Wang, L.; Yuan, Y.; Mechefske, C.K. Relationship between chatter marks and rolling force fluctuation for twenty-high roll mill. Eng. Fail. Anal. 2015, 55, 87-99. [CrossRef]

4. Zhu, Y.; Jiang, W.L.; Kong, X.D.; Wang, H.N. Analytical solution for nonlinear vertical vibration model of mill roll system based on improved complexification averaging method. J. Vibroeng. 2016, 18, 5521-5536.

5. Xue, Z.H.; Cao, X.; Wang, T.Z. Vibration test and analysis on the centrifugal pump. J. Drain. Irrig. Mach. Eng. 2018, 36, 472-477.

6. Zhong, J.; Tang, H.P. Vibration problems of high speed rolling mill-study of dynamics of complex electromechanically coupled system. J. Vib. Meas. Diagn. 2002, 22, 1-8.

7. Wang, Y.Q.; Sun, F.; Liu, J.; Sun, M.H.; Xie, Y.H. Application of smith predictor based on single neural network in cold rolling shape control. Chin. J. Mech. Eng. 2009, 22, 282-286. [CrossRef]

8. Chen, Y.H.; Shi, T.L.; Yang, S.Z. Study on parametrically excited nonlinear vibrations on 4-H cold rolling mills. Chin. J. Mech. Eng. 2003, 39, 56-60. [CrossRef]

9. Sun, J.L.; Peng, Y.; Liu, H.M. Vertical vibration of moving strip in rolling process based on beam theory. Chin. J. Mech. Eng. 2009, 22, 680-687. [CrossRef] 
10. Liu, B.; Jiang, J.H.; Liu, F.; Northeastern University at Qinhuangdao; Institute of Information Technology and Engineering; Yanshan University. Nonlinear vibration characteristic of strip mill under the coupling effect of roll-rolled piece. J. Vibroengineering 2016, 18, 5492-5505. [CrossRef]

11. Liu, H.R.; Shi, P.M.; Chen, H.; Hou, D.X. Study on nonlinear parametrically exited coupling vibrations of roller system on 4-H rolling mills. China Mech. Eng. 2011, 22, 1397-1401.

12. Yan, X.Q. Machinery-electric-hydraulic coupong vibration control of hot continuous rolling mills. Chin. J. Mech. Eng. 2011, 47, 61-65. [CrossRef]

13. Tang, S.N.; Zhu, Y.; Li, W.; Cai, J.X. Status and prospect of research in preprocessing methods for measured signals in mechanical systems. J. Drain. Irrig. Mach. Eng. 2019, 37, 822-828.

14. Zhu, Y.; Tang, S.N.; Quan, L.X.; Jiang, W.L.; Zhou, L. Extraction method for signal effective component based on extreme-point symmetric mode decomposition and Kullback-Leibler divergence. J. Braz. Soc. Mech. Sci. Eng. 2019, 41, 100. [CrossRef]

15. Yang, X.; Li, J.Y.; Tong, C.N. Nonlinear vibration modeling and stability analysis of vertical roller system in cold rolling mill. J. Vib. Meas. Diagn. 2013, 33, 302-306.

16. Bi, Q.S.; Ma, R.; Zhang, Z.D. Bifurcation mechanism of the bursting oscillations in periodically excited dynamical system with two time scales. Nonlinear Dyn. 2015, 79, 101-110. [CrossRef]

17. Bi, Q.S.; Li, S.L.; Kurths, J.; Zhang, Z.D. The mechanism of bursting oscillations with different codimensional bifurcations and nonlinear structures. Nonlinear Dyn. 2016, 85, 993-1005. [CrossRef]

18. Wang, C.; He, X.; Shi, W.; Wang, X.; Wang, X.; Qiu, N. Numerical study on pressure fluctuation of a multistage centrifugal pump based on whole flow field. AIP Adv. 2019, 9, 035118. [CrossRef]

19. Wang, C.; Hu, B.; Zhu, Y.; Wang, X.; Luo, C.; Cheng, L. Numerical study on the gas-water two-phase flow in the self-priming process of self-priming centrifugal pump. Processes 2019, 7, 330. [CrossRef]

20. Wang, C.; He, X.; Zhang, D.; Hu, B.; Shi, W. Numerical and experimental study of the self-priming process of a multistage self-priming centrifugal pump. Int. J. Energy Res. 2019, 43, 4074-4092. [CrossRef]

21. Wang, C.; Shi, W.; Wang, X.; Jiang, X.; Yang, Y.; Li, W.; Zhou, L. Optimal design of multistage centrifugal pump based on the combined energy loss model and computational fluid dynamics. Appl. Energy 2017, 187, 10-26. [CrossRef]

22. He, X.; Jiao, W.; Wang, C.; Cao, W. Influence of surface roughness on the pump performance based on Computational Fluid Dynamics. IEEE Access 2019, 7, 105331-105341. [CrossRef]

23. Wang, C.; Chen, X.X.; Qiu, N.; Zhu, Y.; Shi, W.D. Numerical and experimental study on the pressure fluctuation, vibration, and noise of multistage pump with radial diffuser. J. Braz. Soc. Mech. Sci. Eng. 2018, 40, 481. [CrossRef]

24. Qian, J.Y.; Chen, M.R.; Liu, X.L.; Jin, Z.J. A numerical investigation of the flow of nanofluids through a micro Tesla valve. J. Zhejiang Univ. Sci. A 2019, 20, 50-60. [CrossRef]

25. Qian, J.Y.; Gao, Z.X.; Liu, B.Z.; Jin, Z.J. Parametric study on fluid dynamics of pilot-control angle globe valve. Asme J. Fluids Eng. 2018, 140, 111103. [CrossRef]

26. Bai, L.; Zhou, L.; Jiang, X.P.; Pang, Q.L.; Ye, D.X. Vibration in a multistage centrifugal pump under varied conditions. Shock Vib. 2019, 2019, 2057031. [CrossRef]

27. Bai, L.; Zhou, L.; Han, C.; Zhu, Y.; Shi, W.D. Numerical study of pressure fluctuation and unsteady flow in a centrifugal pump. Processes 2019, 7, 354. [CrossRef]

28. Wang, L.; Liu, H.L.; Wang, K.; Zhou, L.; Jiang, X.P.; Li, Y. Numerical simulation of the sound field of a five-stage centrifugal pump with different turbulence models. Water 2019, 11, 1777. [CrossRef]

29. Ye, S.G.; Zhang, J.H.; Xu, B.; Zhu, S.Q. Theoretical investigation of the contributions of the excitation forces to the vibration of an axial piston pump. Mech. Syst. Signal Process. 2019, 129, 201-217. [CrossRef]

30. Zhang, J.H.; Xia, S.; Ye, S.; Xu, B.; Song, W.; Zhu, S.; Xiang, J. Experimental investigation on the noise reduction of an axial piston pump using free-layer damping material treatment. Appl. Acoust. 2018, 139, 1-7. [CrossRef]

31. Pei, J.; Zhang, F.; Appiah, D.; Hu, B.; Asomani, S.N. Performance prediction based on effects of wrapping angle of a side channel pump. Energies 2019, 12, 139. [CrossRef]

32. Niziol, J.; Światoniowski, A. Numerical analysis of the vertical vibrations of rolling mills and their negative effect on the sheet quality. J. Mater. Process. Technol. 2005, 162-163, 546-550. [CrossRef]

33. Heidari, A.; Forouzan, M.R.; Akbarzadeh, S. Development of a rolling chatter model considering unsteady lubrication. ISIJ Int. 2014, 54, 165-170. [CrossRef] 
34. Heidari, A.; Forouzan, M.R.; Akbarzadeh, S. Effect of friction on tandem cold rolling mills chattering. ISIJ Int. 2014, 54, 2349-2356. [CrossRef]

35. Drzymala, Z.; Świątoniowski, A.; Bar, A. Non-linear vibrations in cold rolling mills. Mécanique Ind. 2003, 4, 151-158. [CrossRef]

36. Xu, H.; Cui, L.L.; Shang, D.G. A study of nonlinear coupling dynamic characteristics of the cold rolling mill system under different rolling parameters. Adv. Mech. Eng. 2017, 9, 1-15. [CrossRef]

37. Wang, Q.L.; Li, X.; Hu, Y.J.; Sun, J.; Zhang, D.H. Numerical analysis of intermediate roll shifting-induced rigidity characteristics of UCM cold rolling mill. Steel Res. Int. 2018, 89, 1700454. [CrossRef]

38. Chen, T.U.; Liu, X.; Gong, Y. Research on fatigue failure of agc servo hydraulic cylinder. Mach. Tool Hydraul. 2017, 45, 158-161.

39. Mosayebi, M.; Zarrinkolah, F.; Farmanesh, K. Calculation of stiffness parameters and vibration analysis of a cold rolling mill stand. Int. J. Adv. Manuf. Technol. 2017, 91, 1-11. [CrossRef]

40. Zhu, Y.; Jiang, W.L.; Kong, X.D.; Zheng, Z. Study on nonlinear dynamics characteristics of electro-hydraulic servo system. Nonlinear Dyn. 2015, 80, 723-737. [CrossRef]

41. Khalid, M.S.U.; Imran, A.; Dong, H.; Ahsan, N.; Wu, B. Bifurcations and route to chaos for flow over an oscillating airfoil. J. Fluids Struct. 2018, 80C, 262-274. [CrossRef]

42. Yu, Y.; Zhang, C.; Han, X.J.; Bi, Q.S. Dynamical behavior analysis and bifurcation mechanism of a new 3-D nonlinear periodic switching system. Nonlinear Dyn. 2013, 73, 1873-1881. [CrossRef]

43. Zhu, Y.; Qian, P.F.; Tang, S.N.; Jiang, W.L.; Li, W.; Zhao, J.H. Amplitude-frequency characteristics analysis for vertical vibration of hydraulic AGC system under nonlinear action. AIP Adv. 2019, 9, 035019. [CrossRef]

44. Liu, F.; Liu, B.; Shi, P.M.; Hou, D.X. Vibration behavior of roll system under nonlinear constraints of the hydraulic cylinder. J. Mech. Eng. 2014, 50, 59-65. [CrossRef]

45. Ling, Q.H.; Yan, X.Q.; Zhang, Q.D.; Zhang, Y.F. Research on vibration characteristics of the hot rolling mill by dual power source driven. J. Vib. Meas. Diagn. 2014, 34, 534-538.

46. Liu, B.; Li, P.; Liu, F.; Liu, H.R.; Jiang, J.H. Vibration behavior and control of roll system under nonlinear stiffness of a hydraulic cylinder. China Mech. Eng. 2016, 27, 3190-3196.

47. Xuan, B.T.; Hafizah, N.; Yanada, H. Modeling of dynamic friction behaviors of hydraulic cylinders. Mechatronics 2012, 22, 65-75.

48. Pennestrì, E.; Rossi, V.; Salvini, P.; Valentini, P.P. Review and comparison of dry friction force models. Nonlinear Dyn. 2016, 83, 1785-1801.

49. Salahshoor, E.; Ebrahimi, S.; Maasoomi, M. Nonlinear vibration analysis of mechanical systems with multiple joint clearances using the method of multiple scales. Mech. Mach. Theory 2016, 105, 495-509. [CrossRef]

50. Zhang, Z.D.; Liu, B.B.; Bi, Q.S. Non-smooth bifurcations on the bursting oscillations in a dynamic system with two timescales. Nonlinear Dyn. 2015, 79, 195-203. [CrossRef]

51. Cirillo, G.I.; Habib, G.; Kerschen, G.; Sepulchre, R. Analysis and design of nonlinear resonances via singularity theory. J. Sound Vib. 2017, 392, 295-306. [CrossRef]

52. Zhang, C.; Han, X.J.; Bi, Q.S. On symmetry-breaking bifurcation in the periodic parameter-switching Lorenz oscillator. Sci. China-Technol. Sci. 2013, 56, 2310-2316. [CrossRef]

53. Zhang, R.; Wang, Y.; Zhang, Z.D.; Bi, Q.S. Nonlinear behaviors as well as the bifurcation mechanism in switched dynamical systems. Nonlinear Dyn. 2015, 79, 465-471. [CrossRef]

54. Wu, Z.Q.; Yu, P.; Wang, K.Q. Bifurcation analysis on a self-excited hysteretic system. Int. J. Bifurc. Chaos 2004, 14, 2825-2842. [CrossRef]

(C) 2019 by the authors. Licensee MDPI, Basel, Switzerland. This article is an open access article distributed under the terms and conditions of the Creative Commons Attribution (CC BY) license (http://creativecommons.org/licenses/by/4.0/). 